\title{
AM CVn SYSTEMS AS OPTICAL, X-RAY AND GWR SOURCES
}

\author{
L. Yungelson, ${ }^{1}$ G. Nelemans, ${ }^{2}$ and S. F. Portegies $Z$ wart ${ }^{3}$
}

\begin{abstract}
RESUMEN
Discutimos el modelo para la muestra Galáctica de sistemas AM CVn con $P_{\text {orb }} \leq 1500$ s detectables en la banda óptica y/o en rayos-X y de resolverse por el detector de ondas gravitacionales LISA. En el caso de $3 \lesssim P \lesssim 10$ min todos los sistemas que se detectan son fuentes de rayos-X. En ei caso de la $P \gtrsim 10$ min mayoría de los sistemas se detectan sólo en la banda V. Alrededor de 10,000 sistemas AM CVn pueden ser resueltos por LISA; esto es comparable con el número de enanas blancas dobles separadas que pueden ser resueltas. Es posible que varios cientos de fuentes AM CVn de LISA puedan ser también detectadas en las bandas V y/o de rayos-X.
\end{abstract}

\section{ABSTRACT}

We discuss the model for the Galactic sample of the AM CVn systems with $P_{\text {orb }} \leq 1500 \mathrm{~s}$ that can be detected in the optical and/or X-ray bands and may be resolved by the gravitational waves detector LISA. At $3 \lesssim P \lesssim 10 \mathrm{~min}$ all detectable systems are X-ray sources. At $P \gtrsim 10 \mathrm{~min}$ most systems are only detectable in the $V$-band. About $30 \%$ of the X-ray sources is also detectable in the $V$-band. About 10,000 AM CVn systems might be resolved by LISA; this is comparable to the number of detached double white dwarfs that can be resolved. Several hundreds of AM CVn LISA sources might be also detectable in the $V$ - and/or X-ray bands.

Key Words: BINARIES: CLOSE - GRAVITATIONAL WAVES - WHITE DWARFS

\section{INTRODUCTION}

AM CVn stars are short-period binaries $(P \lesssim$ $1 \mathrm{hr}$ ) that are observed at optical wavelengths as faint blue and variable objects. Their optical variability is of dwarf novae or nova-like type. Some AM CVn stars are also observed as X-ray sources. Currently, the class includes 13 confirmed and candidate objects. Note, that two candidates have the shortest orbital periods and were first discovered as $\mathrm{X}$-ray sources. Observations of AM CVn systems were recently reviewed, e.g., by Solheim (2003). A discussion of the model of the population of AM CVn stars can be found in Nelemans, Yungelson, \& Portegies Zwart (2003).

AM CVn stars are thought to be semidetached binaries with both components being white dwarfs; evolution of the system is driven by the loss of orbital angular momentum via radiation of gravitational waves (Paczyński 1967). The total Galactic number of AM CVn stars is estimated as $\sim 3.7 \cdot 10^{7}$ (Nelemans et al. 2003), but the state of understanding of their formation and uncertainties in, e.g., the star formation history, the initial mass function of stars and the parameters entering population synthesis make this estimate uncertain by a factor $\sim 10$

\footnotetext{
${ }^{1}$ Institute of Astronomy, RAS, Moscow, Russia.

${ }^{2}$ Institute of Astronomy, U. Cambridge, UK.

${ }^{3} \mathrm{U}$. Amsierdam, the Netherlands.
}

(Nelemans, Portegies Zwart, Verbunt, \& Yungelson 2001; Nelemans et, al. 2003).

We consider the model for the subset of shortest period AM CVn stars ( $P \leq 1500$ s) that could be detected by the gravitational wave (GWR) detector LISA and focus on the systems that may have optical and/or X-ray counterparts. At $P \gtrsim 1500$ s AM CVn stars do not emit interesting amounts of energy in the GW band and optical radiation from the disc completely dominates emission of the star.

The model of the population of AM CVn stars is obtained by population synthesis code SeBa (Portegies Zwart \& Verbunt 1996; Nelemans et al. 2003). The results we discuss represent one possible random realization of the model, so all numbers given are subject to Poisson noise.

\section{OPTICAL, X-RAY AND GW EMISSION OF AM CVn STARS}

Some of the AM CVn stars, are so compact that they are in the "direct impact" phase when the accretion disk is absent. Thus, there are four emission sites: the accretion disc, the impact spot in the direct impact accretors, the donor and the accretor.

If the disc is present, we assume that half of the energy released by accretion is radiated by the disc in the optical band, while the rest is emitted as solt $\mathrm{X}$-rays from the boundary layer. For the sitimate of optical emission we apply a single-temperature 


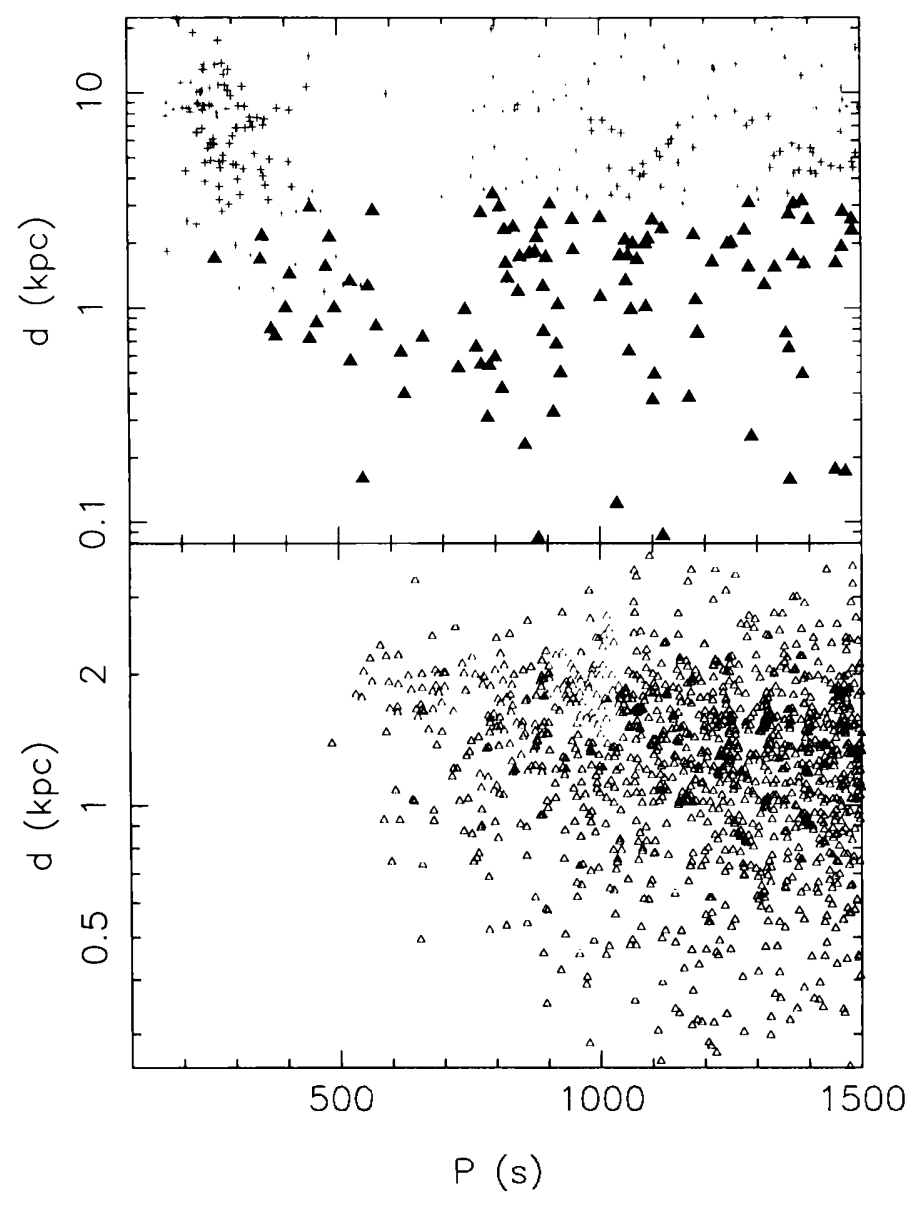

Fig. 1. Top panel: Period vs, distance distribution of systems detectable only in X-rays (pluses), and systems that are detectable in the optical and X-ray band (filled triangles). Bottom pancl: Systems only detectable in the optical band.

disc model (Wade 1984). For the X-ray emission of boundary layer we use the model of Pringle (1977).

For direct impact systems we assume that whole accretion luminosity is radiated as a blackbody within the fraction $f=0.001$ of the total surface of the dwarf (Marsh \& Steeghs 2002).

Emission of the donor and accretor comes from the cooling which we approximate using models of Hansen (1999). Wo neglect irradiation effects and tidal or compressional heating.

Interstellar reddening is taken into account using the Sandage (1972) model. For the estimate of absorption in X-rays, hydrogen column density is modelled after Predehl \& Schmitt (1995).

We assume that AM CVn systems emit GW as point sources. The strain amplitude is evaluated by standard formalism (e.g.. Evans, Iben, \& Smarr 1987).

\section{RESULTS}

In Figure 1 we show the model sample of the systems that would be detectable in the optical and/or
X-ray bands. We limit the "optical" sample by $V_{\text {lim }}=20 \mathrm{mag}$, which is close to the $V_{\text {lim }}$ of observed AM CVn stars. The "X-ray" sample is comprised by the systems that have a flux in the $0.1-2.4 \mathrm{keV}$ (ROSAT) band higher than $10^{-13} \mathrm{erg} \mathrm{s}^{-1} \mathrm{~cm}^{-2}$.

The top panel of Fig. 1 shows 220 systems detectable in X-rays only (pluses) and the additional 106 systems that are also detectable in the $V$-band (filled triangles). There are 75 systems with optical emission from a disc and 28 systems close to the Earth, in which the donor stars can be seen as well as the discs. About half of X-ray systems are in the direct impact phase. Note, at the shortest periods a significant fraction of X-ray systems are detectable even close to the Galactic centre.

The bottom panel of Fig. 1 shows 1230 "conventional" AM CVn systems, detectable only by the optical emission, mainly from their accretion disc. Of this population 169 ones closest to the Earth also have a visible donor. The "optical" systems with periods betwen 1000 and $1500 \mathrm{~s}$ are expected to have unstable discs (Tsugawa \& Osaki 1997); this may enhance the probability of their discovery.

Figure 1 clearly predicts the existence of a significant population of systems that might be detectable only as X-ray sources. Some have periods as short as three minutes. About one third of the X-ray sources is also detectable in the optical band. This prediction is sustained by discovery of sources that are detectable in X-rays and (just) in the optical band.

Previous studies of GW emission of the AM CVn systems (e.g. Hils \& Bender 2000; Nelemans, Yungelson, \& Portegies Zwart 2001) have found that they hardly contribute to the GW background noise, even though at $\nu=0.3-1 \mathrm{mHz}$ they outnumber the detached double white dwarfs. This is because at these $\nu$ their chirp mass $\mathcal{M}$ is well below that of a typical detached system. But it was overlooked that at higher $\nu$, where the number of AM CVn systems is much smaller, their $\mathcal{M}$ is similar to that of the detached systems from which they descend. Our model shows that, out of the total population of $\sim 140,000$ AM CVn stars with $P \leq 1500$ s, for $T_{\mathrm{obs}}=1 \mathrm{yr}, L I S A$ may be expected to resolve $\sim 11,000$ double white dwarfs at $S / N \geq 5$ as well as $\sim 11,000 \mathrm{AM} C V n$ systems at $S / N \geq 1$ (or $\sim 3000$ at $S / N \geq 5$ ).

In Figure 2 we show the distributions vs. orbital periods for the total number of AM CVn systems with $P \leq 1500$ s and for AM CVn- $L I S A$ sources that have optical and/or X-ray counterparts, direct impact systems that are GWR and X-ray sources, and the total number of systems that are optical and/or 


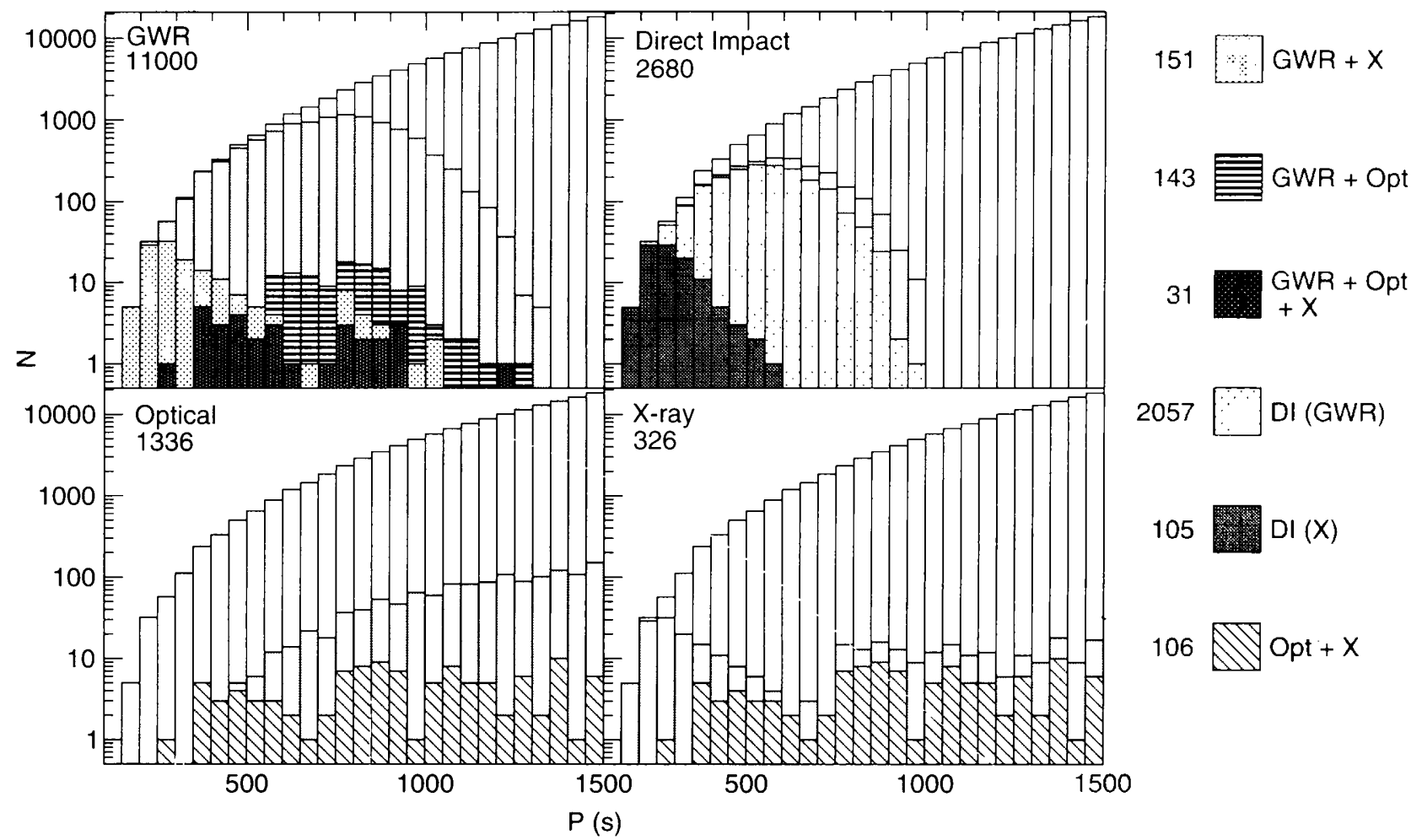

Fig. 2. Short-period AM CVn systems, subdivided in different types. Each panel shows the total population as the white histogram. The top left panel shows the systems that can be resolved by LISA in grey and they are subdivided in the ones that have optical counterparts (GWR + Opt), X-ray counterparts (GWR + X) and both (GWR + Opt + $\mathrm{X}$ ). The top right panel shows the systems that are in the direct impact phase of accretion in grey and it is subdivided in GWR and X-ray sources. The bottom two panels show (again in grey) the populations that are detectable in the optical band (left panel) and the X-ray band (right panel). The distribution of sources detectable both in optical and $\mathrm{X}$-ray bands is shown as hatched bins in both lower panels $(\mathrm{Opt}+\mathrm{X})$.

$\mathrm{X}$-ray sources. The interrelations between numbers of sources emitting in different wavebands are shown in the legend at the right of the Figure.

It is clear from Fig. 2 that LISA is expected to be very effective in detecting the short-period AM CVn systems. About $20 \%$ of them will be in the direct impact phase and at short periods might be detectable in X-rays. Towards longer periods the systems detectable in the optical band take over.

The GW signal from a monochromatic binary is characterized by seven independent parameters. Therefore in the data analysis all parameters, except the amplitude $h$, must be estimated using a fitting technique for which an initial guess is needed (Hellings 2003). Information from observations in the optical and X-ray bands will facilitate these guesses. On the other hand, e.g., inclinations of orbits and chirp masses obtained by GW observations would provide information for the estimates of parameters of AM CVn stars.

L.Y. acknowledges financial support from
CONACYT 34521-E and RFBR 03-06-16254 grants. REFERENCES

Evans, C. R., Iben, I. J., \& Smarr, L. 1987. ApJ, 323, 129

Hansen, B. M. S. 1999, ApJ, 520, 680

Hellings, R. 、v. 2003, Class.Quant.Grav., 20, 1019

Hils, D. \& Bender, P. L. 2000, ApJ, 537, 334

Marsh, T. R. \& Steeghs, D. 2002, MNRAS, 331, L7

Nelemans, G., Portegies Zwart, S. F.. Verbunt, F. \& Yungelson, L. R. 2001, A\&A, 368, 939

Nelemans, G., Yungelson, L. R., \& Portegies Zwart. S. F. 2001, A\&A, 375, 890

-. 2003, MNRAS, in press

Paczyński, B. 1967, Acta Astron., 17. 287

Portegies Zwart, S. F. \& Verbunt, F. 1996. Ad.A. 309. 179

Predehl, P. \& Schmitt, J. H. M. M. 1995, Ad.1. 293. 889

Pringle, J. E. 1977, MNRAS, 178, 195

Sandage, A. 1972, ApJ, 178, 1

Solheim, J.-E. 2003, in NATO ASIB Proc. 105: White Dwarfs, 299

Tsugawa, M. \& Osaki, Y. 1997, P.AS.J, 49, i5

Wade, R. A. 1984, NINAS. 208, 3\$1 